\title{
Classification of Staphylococcus albus strains isolated from the urinary tract
}

\author{
R. G. M I T C H E L L
}

\author{
From the Department of Bacteriology, Royal Infirmary, Bristol
}

SYNOPSIS Strains of Gram-positive, catalase-positive, coagulase-negative cocci isolated from urine specimens were classified by the system of Baird-Parker (1963). Urinary infections attributed to instrumentation or pathological abnormalities of the urinary tract were caused almost entirely by various Staphylococcus subgroups. These showed a wide range of antibiotic sensitivities, unrelated to subgroups and not obviously related to previous chemotherapy. Attacks of cystitis occurring in otherwise healthy women were usually caused by Micrococcus subgroup 3 strains.

During an investigation of urinary tract infections caused by Staph. albus (Mitchell, 1964), it was noticed that strains isolated from different patients could vary in their colonial appearances and antibiotic sensitivities. It was not known whether infections were caused by a wide range of coagulasenegative biotypes encountered as commensals on human skin or mucous membranes (Baird-Parker, 1962), or by a limited number of more pathogenic types; nor was it known whether the varied antibiotic sensitivities were due to type differences or to modification as the result of previous antibiotic treatment. It was therefore decided to extend the survey and study subsequent isolates in greater detail.

\section{MATERIALS AND METHODS}

Specimens of urine were received from hospital wards and outpatient departments as well as from domiciliary practice. A urinary infection was diagnosed when a midstream or catheter specimen of urine, examined within three hours of collection, showed numerous Grampositive cocci in clusters in the stained deposit without significant numbers of squames, and culture yielded a profuse growth of Staph. albus without other organisms. Bacterial counts were not done in every case, but were never less than $10^{5}$ organisms per $\mathrm{ml}$. and were usually higher. An excess of white cells (more than 10 per highpower field in the centrifuged deposit) was not an essential criterion of infection but was usually present. Cultures usually appeared pure, but when mixed colonial types were found, representative strains were selected for study. The time of collection of a urine specimen was not

Received for publication 26 July 1967.

Present address: Department of Pathology, Churchill Hospital, Oxford. always stated, but if in this event there was no reason to suspect delay in transit to the laboratory, strains were retained from profuse pure growths which indicated the possibility of infection.

The methods described by Baird-Parker (1963) were used to classify strains: organisms which formed acid from glucose anaerobically were assigned to the genus Staphylococcus, and those which produced acid aerobically or not at all to the genus Micrococcus. Strains were allocated to the various Baird-Parker subgroups using the following tests: coagulase, phosphatase and acetoin production, mode of attack on glucose, and acid production from mannitol, lactose, and l-arabinose (Baird-Parker, 1963). For convenience the production of acid from all carbohydrates was studied in 6 in. $\times 1 / 2$ in. tubes of medium instead of using plate cultures. During the latter part of the work, the medium used for carbohydrate utilization was that recommended by the Association of Microbiological Societies'Subcommittee on Staphylococci and Micrococci (Baird-Parker, personal communication).

Antibiotic sensitivities were determined by a simple agar diffusion-disc method, using a Sentest low-potency penicillin tablet, and the following mast discs : streptomycin $25 \mu \mathrm{g}$., chloramphenicol $25 \mu \mathrm{g}$., tetracycline $25 \mu \mathrm{g}$., erythromycin $5 \mu \mathrm{g}$., and novobiocin $5 \mu \mathrm{g}$.

ENUMERATION OF STRAINS The urine cultures studied in this survey were usually pure, and even when apparently mixed were sometimes found to consist of colonial variants of the same subgroup. When on occasion more than one subgroup was present in a culture, each was enumerated separately. The same subgroup was frequently isolated from serial urine specimens obtained from the same patient, and if so was enumerated once only. If such strains altered in their antibiotic sensitivities, only the most resistant pattern was recorded If successive unrelated urinary infections occurred in the same patient, the organisms isolated were enumerated 
separately. Where possible, each infection was placed into a single clinical category, but this was not always easy or indeed possible when several likely causal factors were operating simultaneously.

\section{RESULTS}

One hundred and forty-seven strains of Staph. albus, isolated from 138 patients, were classified into various Baird-Parker subgroups. The clinical categories of the patients and the corresponding subgroups of the strains are shown in Table $I$. Urinary infections following urethral instrumentation were caused almost entirely by various Staphylococcus subgroups. Staphylococcus subgroup II strains greatly predominated, followed in order of frequency by subgroups V, VI, and IV. The sole exception was a Micrococcus subgroup 3 strain, isolated from a woman who had undergone a gynaecological repair operation and was subsequently catheterised. In the second group of patients with pathological and anatomical abnormalities of the urinary tract, Staphylococcus subgroups again predominated, mostly subgroup II, but Micrococcus subgroup 3 strains were isolated from three patients with urinary calculi. In the third group of 'primary' infections, Micrococcus subgroup 3 strains predominated, and this was almost invariably the biotype of strains causing cystitis and acute pyelonephritis in domiciliary practice. Sixty-five of the total strains were isolated from urine specimens known to be $i$ less than three hours old when cultured. Their clinical distribution, shown in Table $I$, is representative of the series as a whole, suggesting that many of the

TABLE I

CORRELATION OF CLINICAL CATEGORIES WITH BIOTYPES OF STRAINS OF STAPH. ALBUS ISOLATED FROM 138 PATIENTS

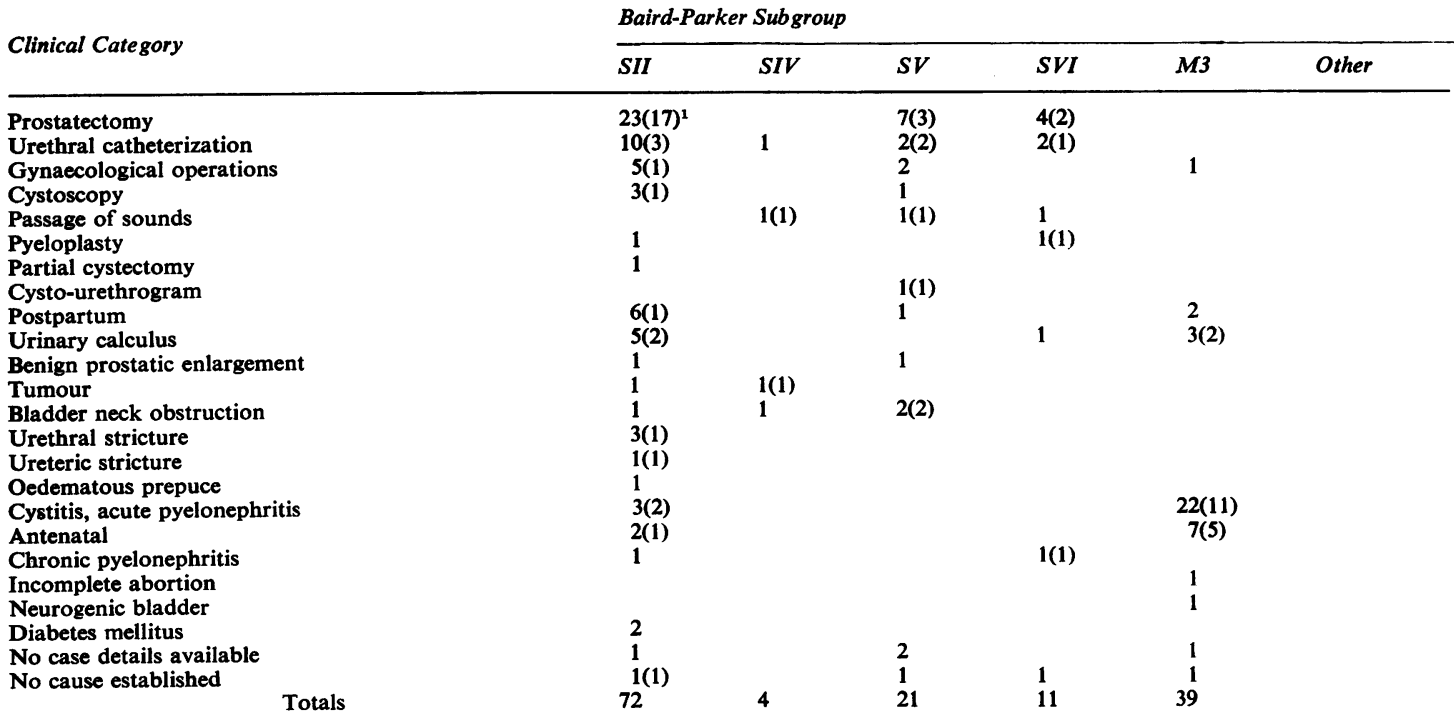

${ }^{1}$ Numbers in parentheses denote strains isolated from urine specimens known to be less than three hours old (total 65 ).

TABLE II

ANTIBIOTIC RESISTANCE PATTERNS OF STAPHYLOCOCCUS AND MICROCOCCUS SUBGROUPS ISOLATED FROM URINE SPECIMENS Resistant to:

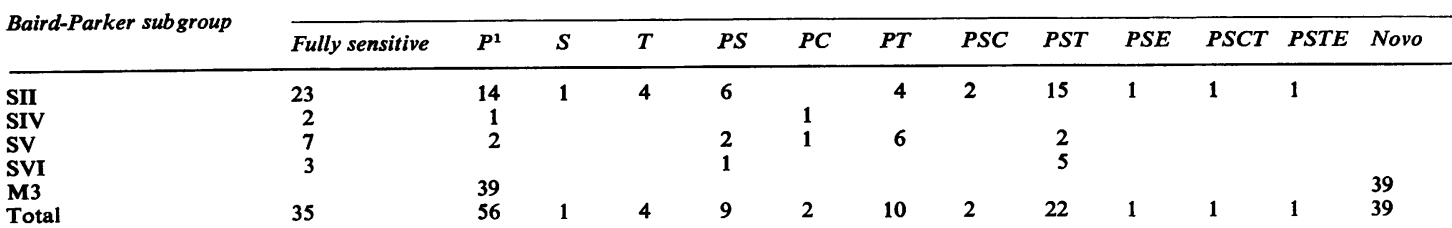

iP denotes resistance to penicillin, $\mathbf{S}$ to streptomycin, $\mathrm{C}$ to chloramphenicol, $\mathrm{T}$ to tetracycline, $\mathrm{E}$ to erythromycin, Novo to novobiocin. 
TABLE III

CORRELATION OF STAPHYLOCOCCUS SUBGROUPS WITH CHEMOTHERAPY RECEIVED DURING ONE MONTH BEFORE ISOLATION OF STRAINS

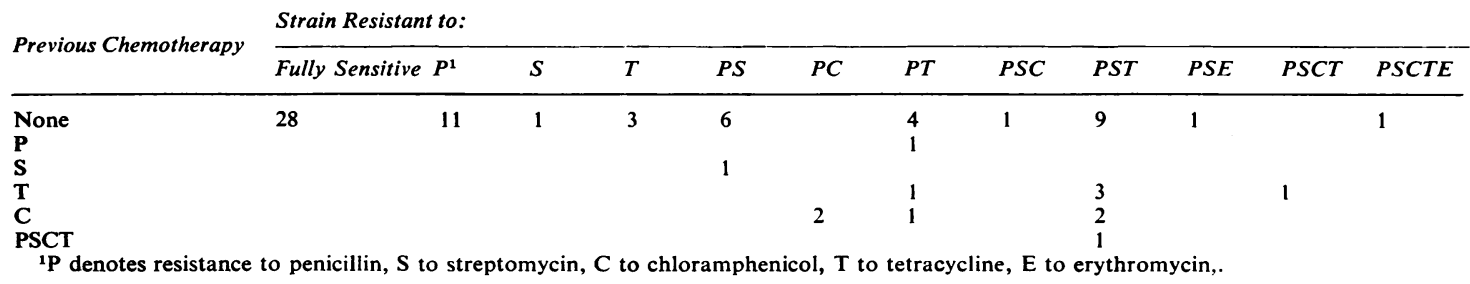

possible' infections were in fact 'true' infections.

Staph. albus strains showed a wide range of antibiotic sensitivities. These are shown with the subgroups in Table II. Thirty-five of the 105 Staphylococcus strains, which included most of the strains isolated after instrumentation, were fully sensitive to penicillin, streptomycin, chloramphenicol, tetracycline, and erythromycin. Next most commonly isolated were strains resistant to penicillin, streptomycin, and tetracycline, followed by strains resistant to penicillin only.

Antibiotic sensitivities did not appear to be related in any way to particular Staphylococcus subgroups. All the Staphylococcus strains studied were sensitive to novobiocin. In contrast, all 39 Micrococcus subgroup 3 strains were resistant to penicillin and novobiocin, and sensitive to other common antibiotics tested.

Since previous chemotherapy might have contributed to the resistance pattern of strains, details were sought of any antibiotic therapy received by the patient within the month preceding isolation of the organism. It could not always be established whether any drugs had been administered by the family doctors before the patients were admitted to hospital. The available details of treatment are related to the antibiotic resistance patterns in Table III. Twenty-eight of the 35 patients with fully sensitive Staphylococcus strains were not known to have received antibiotics. However, 37 of the 70 patients yielding Staphylococcus strains resistant to at least one antibiotic had probably not received antibiotic treatment, including nine of 22 patients with strains which showed multiple resistance to penicillin, streptomycin, and tetracycline. At least two out of five patients with chloramphenicolresistant strains had received chloramphenicol in the previous month; yet chloramphenicol had been administered to at least four patients who later yielded chloramphenicol-sensitive strains.

\section{DISCUSSION}

Gallagher, Montgomerie, and North (1965) found that coagulase-negative staphylococci were the second commonest causal organism in uncomplicated urinary tract infections of women in general practice, and drew attention to the confused nomenclature of this group of organisms. The system of classification proposed by Baird-Parker (1963) has been successfully adapted in this study to clinical investigation. It is based on biochemical tests which are well within the scope of a hospital laboratory and achieves considerable discrimination between strains, which makes it possible to follow the natural history of infections by these organisms.

The majority of infections in this series, including practically all those acquired in hospital, were caused by a variety of Staphylococcus subgroups with very varied antibiotic sensitivities. These infections either followed instrumentation of the urinary tract, notably prostatectomy, or were superimposed on preexisting lesions of the urinary tract. It appears, therefore, that coagulase-negative Staphylococcus subgroups can act as relatively feeble 'secondary' pathogens which require a nidus of damaged tissue before they can become established.

Modification of antibiotic sensitivities by previous chemotherapy may have occurred, but was not convincingly shown in this investigation. However, a period of one month before the isolation of strains was possibly not long enough in which to observe the effects of chemotherapy. It was found for instance that a patient whose urine yielded a chloramphenicol-resistant strain in the July of one year had not received treatment with chloramphenicol since the previous December.

Micrococcus subgroup 3 strains caused urinary tract infections in 39 patients (33 females and six males). With one exception, these occurred in domiciliary practice, and the majority of patients were married women with cystitis or acute pyelonephritis, in some of whom infections were related to intercourse. Micrococcus subgroup 3 strains could be provisionally identified by their resistance to novobiocin. Further work arising from this study has shown that all glucose-oxidizing Micrococcus subgroups are resistant to novobiocin (Mitchell and 
Baird-Parker, 1967). For this purpose novobiocin sensitivity is being determined solely as an indicator of difference between 'wild' strains of Staphylococci and Micrococci. It is appreciated that coagulasenegative Staphylococci may perhaps acquire resistance to novobiocin, but one would not expect this drug to be used in the treatment of urinary infections, since, it is not excreted in large amounts in the urine (Wright, Putnam, and Welch, 1956). Torres Pereira (1962) described a series of 40 cases of urinary tract infections in women caused by strains of Staph. albus which were serotypically homogeneous and possessed a common antigen 51 . Strains from two of these patients, kindly provided by Professor Pereira, were classified as Micrococcus subgroup 3.

Our findings suggest that Micrococcus subgroup 3 is a 'primary' pathogen in the sense that it can cause infections, sometimes of considerable severity, in otherwise healthy subjects who have no demonstrable abnormalities of the urinary tract. A few of the strains isolated in the present study have been shown to possess ribitol teichoic acid in their cell walls (Dr. A. L. Davison, personal communication), in common with coagulase-positive Staph. aureus strains (Davison and Baddiley, 1963). Micrococcus subgroup 3 is therefore analogous to the inter- mediate' staphylococcus of Losnegaard and Oeding $\stackrel{\overrightarrow{0}}{\overrightarrow{0}}$ (1963) and it may in fact owe its pathogenicity to the presence of ribitol teichoic acid acting as an anti- $\stackrel{\oplus}{\rightarrow}$ genic determinant. If so, it is curious that attacks of 0 cystitis occurring in domiciliary practice are so seldom caused by Staph. aureus, but this probably $\frac{\sigma}{7}$ reflects the preponderance of coagulase-negative $\stackrel{\mathbb{Q}}{\Omega}$ over coagulase-positive cocci present as commensals in the urethra (Clabaugh and Rhoads, 1957).

I am indebted to Dr. A. L. Davison for examining some of the above strains, to Dr. A. C. Baird-Parker for his ${ }_{0}$ constant help and advice, to Professor A. Torres Pereira $\overline{ }$ for providing me with strains from his collection, and to Professor W. A. Gillespie, under whose direction this work was carried out.

\section{REFERENCES}

Baird-Parker, A. C. (1962). J. appl. Bact., 25, 352.

- (1963). J.gen. Microbiol., 30, 409.

Clabaugh, G. F., and Rhoads, P. S. (1957). J. Amer. med. Ass., 165, 815.

Davison, A. L., and Baddiley, J. (1963). Ibid., 32, 271.

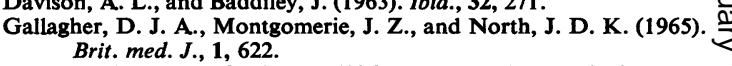

Losnegaard, N., and Oeding, P. (1963). Acta path. microbiol. scand., 58, 493.

Mitchell, R. G. (1964). J. clin. Path., 17, 105.

-, and Baird-Parker, A. C. (1967). J. appl. Bact., 30, 251.

Torres Pereira, A. (1962). J. clin. Path., 15, 252.

Wright, W. W., Putnam, L. E., and Welch, H. (1956). Antibiot. Med., 2,311 . 